\title{
Evaluación de ozono ambiental en el municipio de Mineral de la Reforma, Hidalgo
}

\author{
Cristian Alain Briones Vázqueza ${ }^{\mathrm{a}}$, María Guadalupe Moreno Bautista ${ }^{\mathrm{a}}$, Vivian Itzel Moreno Ramírez ${ }^{\mathrm{a}}$, Rosa Icela Beltrán \\ Hernández ${ }^{\mathrm{a}}$, Carlos Alexander Lucho Constantino ${ }^{\mathrm{a}, *}$
}

a Área Académica de Química, Licenciatura en Química, Universidad Autónoma del Estado de Hidalgo. Carretera Pachuca-Tulancingo km. 4.5, Colonia Carboneras. Mineral de la Reforma, Hidalgo. México CP. 42184.

\section{Resumen}

El ozono $\left(\mathrm{O}_{3}\right)$ se encuentra presente en distintas capas de la atmósfera: en la estratosfera, actúa como filtro de las radiaciones ultravioleta emitidas por el sol, mientras que en la troposfera es un contaminante dañino para los organismos vivos y también es un gas precursor de efecto invernadero. El objetivo de este estudio fue evaluar las variaciones horarias en la concentración de $\mathrm{O}_{3}$ en el municipio de Mineral de la Reforma, Hidalgo, para ello empleamos detectores colorimétricos preparados con una solución de almidón/KI. Los resultados obtenidos por la exposición de los detectores al $\mathrm{O}_{3}$ en la zona de estudio mostraron que la máxima concentración de este gas se presentó a las $12 \mathrm{~h} 00$, con una temperatura promedio de $18.5^{\circ} \mathrm{C}$ y una humedad relativa entre 16 y $20 \%$.

Palabras Clave: detector de ozono, calidad del aire, contaminante secundario, salud.

\section{Introducción}

En la actualidad, el creciente desarrollo industrial y el incremento del flujo vehicular han contribuido al deterioro de la calidad del aire de ciudades y áreas rurales, lo cual a su vez ha provocado diversos problemas de salud en la población. La contaminación atmosférica se presenta por un desequilibrio en las concentraciones de los componentes del aire y se favorece por la presencia cada vez mayor de los contaminantes (NOM020-SSA1-2014).

Los contaminantes atmosféricos se clasifican por su origen en primarios y secundarios. Los contaminantes primarios son los que se encuentran en la atmósfera tal y como fueron generados, ya sea por procesos naturales o por actividades antropogénicas. Algunos ejemplos de estos el monóxido de carbono (CO), dióxido de azufre $\left(\mathrm{SO}_{2}\right)$ y dióxido de nitrógeno $\left(\mathrm{NO}_{2}\right)$. Los contaminantes secundarios son aquellos que se forman en la atmósfera por reacciones químicas o fotoquímicas entre los contaminantes primarios o entre estos y los componentes de la atmósfera, el ozono $\left(\mathrm{O}_{3}\right)$ y los sulfatos son ejemplos de ellos.

Los contaminantes que se monitorean rutinariamente en el aire son el $\mathrm{CO}$, el $\mathrm{SO}_{2}$, el $\mathrm{NO}_{2}$, el plomo $(\mathrm{Pb})$, las partículas suspendidas totales (PST), las partículas menores de 10 micrómetros de diámetro $\left(\mathrm{PM}_{10}\right)$ y el $\mathrm{O}_{3}$ (Vallejo et al., 2003). A los contaminantes anteriores se les ha llamado contaminantes criterio, por ser los más comúnmente emitidos por las actividades humanas, y desde luego por su potencial para afectar la salud y bien de la población.

$\mathrm{El} \mathrm{O}_{3}$ es un contaminante secundario que se forma por una reacción fotoquímica entre emisiones primarias de óxidos de nitrógeno (NOx), compuestos orgánicos volátiles (COV) o hidrocarburos (HC) en presencia de la radiación solar. Por ello es común encontrar altos niveles de ozono en el aire en un día soleado, lo cual puede desencadenar ataques de asma y problemas respiratorios en personas susceptibles (Ferrer y

Escalante, 2009). Lo anterior pone de manifiesto la importancia de conocer la concentración de este contaminante en el aire.

$\mathrm{El} \mathrm{O}_{3}$, además de afectar el sistema respiratorio, también puede comprometer el sistema inmunológico y causar problemas dérmicos en los seres humanos.

Los estudios más recientes de la calidad del aire en el estado de Hidalgo los realizó la Secretaría de Medio Ambiente y Recursos Naturales del Estado en el año 2014. (INECC, 2015). El Sistema de Monitoreo Atmosférico del Estado de Hidalgo cuenta con diez estaciones de monitoreo distribuidas en los municipios de Ajacuba, Atitalaquia, Atotonilco, Tepetitlán, Tepeji del Río, Tlaxcoapan, Tizayuca, Lolotla, Tepeapulco y Huichapan.

De acuerdo con los datos reportados por la SEMARNAT, durante el año 2014, el $\mathrm{O}_{3}$ presentó comportamiento unimodal donde las concentraciones máximas se encontraron entre las $12 \mathrm{~h} 00$ y $18 \mathrm{~h} 00$ en la mayoría de las estaciones de monitoreo (INECC, 2015).

En la Tabla 1 se muestran las concentraciones de $\mathrm{O}_{3}$ de las cuatro estaciones de monitoreo que presentaron los niveles más altos de este contaminante: en la estación de Tizayuca se encontró el límite máximo permisible por la NOM-020-SSA11993, mientras que en las de Pachuca y Atotonilco la concentración encontrada sobrepasó este límite. Cabe hacer mención que la norma mexicana establece una concentración máxima permisible de $110 \mathrm{ppb}$ de $\mathrm{O}_{3}$ en un periodo de una hora.

\footnotetext{
* Autor en correspondencia.

Correo electrónico: luchouaeh@gmail.com (Carlos A. Lucho Constantino)
} 
Tabla 1: Estaciones de monitoreo con mayor concentración de $\mathrm{O}_{3}$ en el Estado de Hidalgo.

\begin{tabular}{ccc}
\hline Ciudad & Estación de monitoreo & Concentración de $\mathrm{O}_{3}(\mathrm{ppb})$ \\
\hline Pachuca & Museo del Rehilete & 145 \\
& Tizayuca & 110 \\
Xochicoatlán & Atotonilco & 111 \\
& Atitalaquia & 83
\end{tabular}

Debido a que la estación con la mayor concentración de $\mathrm{O}_{3}$ fue la ubicada en Pachuca, el objetivo de nuestro estudio fue determinar las condiciones ambientales en las que se presentó la concentración máxima de $\mathrm{O}_{3}$ en el ambiente en una zona urbana (Mineral de la Reforma, Hidalgo) cercana a la ciudad de Pachuca, donde no existen unidades de monitoreo atmosférico. La cuantificación de $\mathrm{O}_{3}$ se realizó empleando detectores colorimétricos, en los cuales el ozono reacciona con yoduro de potasio en presencia de agua, liberando yodo molecular que produce una coloración que permite conocer la concentración de ozono atmosférico en un sitio determinado.

\section{Metodología}

\subsection{Sitio de estudio}

La evaluación de $\mathrm{O}_{3}$ ambiental se realizó en la Colonia Carboneras del municipio de Mineral de la Reforma, Hidalgo $\left(20^{\circ} 05^{\prime} 31.6^{\prime \prime} \mathrm{N}, 9^{\circ} 42^{\prime} 35.3^{\prime \prime} \mathrm{O}\right)$, para ello se expusieron dieciséis detectores colorimétricos al ambiente, cada uno por un periodo de una hora. La exposición inició a las 7h00 del 9 de marzo del 2016 y concluyó a las $22 \mathrm{~h} 00$ del mismo día.

Los detectores de $\mathrm{O}_{3}$ fueron elaborados con base en la metodología propuesta en el manual de Laboratorio de Protección Ambiental I, UNAM (2010).

\subsection{Materiales, reactivos y equipo}

En la tabla 1 se presentan los materiales, reactivos y equipos necesarios para realizar la determinación de $\mathrm{O}_{3}$ atmosférico.

Tabla 1. Materiales, reactivos y equipos.

\begin{tabular}{cccc}
\hline Reactivos & Cantidad & Materiales & Equipos \\
\hline Agua destilada & 1 & Vidrio de Reloj & Parrilla con agitación \\
Yoduro de potasio & 1 & Espátula & Balanza digital \\
Almidón & $1 / 4$ & Papel Filtro (pliego) & Estufa de secado \\
$\mathrm{H}_{2} \mathrm{SO}_{4}[1 \mathrm{M}]$ & 2 & Vasos de precipitado de $250 \mathrm{~mL}$ & \\
& 2 & Bolsas de plástico sellables & \\
& 1 & Brocha 1/4 pulgada & \\
& 1 & Agitador de varilla de vidrio & \\
& 1 & Carta psicométrica & \\
\hline
\end{tabular}

\subsection{Preparación de los detectores de $\mathrm{O}_{3}$}

Los detectores de $\mathrm{O}_{3}$ se elaboraron con tiras de papel filtro impregnadas de una solución de almidón y KI de acuerdo al siguiente procedimiento: se disolvieron $5 \mathrm{~g}$ de almidón en 10 $\mathrm{mL}$ de agua fría, posteriormente, se adicionaron $90 \mathrm{~mL}$ de agua caliente y se calentó la solución a ebullición durante 15 minutos. Luego se adicionó $0.5 \mathrm{~g}$ de $\mathrm{KI}$ a la solución de almidón, se agitó y una vez disuelto se adicionó $1 \mathrm{~mL}$ de $\mathrm{H}_{2} \mathrm{SO}_{4} 1 \mathrm{M}$. Posteriormente, se cortaron 20 tiras de $1 \times 5 \mathrm{~cm}$ de papel filtro, las cuales se identificaron con un número $\mathrm{y}$ después se impregnaron con la solución de almidón/KI empleando una brocha. Finalmente se secaron en un horno a $80^{\circ} \mathrm{C}$ durante 4 minutos. Una vez secas las tiras se almacenaron en bolsas de plástico con cierre hermético hasta su uso.

\subsection{Evaluación del Ozono ambiental}

La medición del $\mathrm{O}_{3}$ ambiental se realizó de la siguiente manera: uno de los detectores se expuso durante todo el tiempo de medición: desde las $7 \mathrm{~h} 00$ hasta las $22 \mathrm{~h} 00$. Se cuidó que el detector no recibiera directamente los rayos del sol para evitar interferencias con la reacción de formación del $\mathrm{O}_{3}$. Otro detector se guardó en una bolsa con cierre hermético, este sirvió como control. El resto de los detectores se expusó únicamente durante una hora, al término de ese tiempo eran reemplazados por otro detector, de esta manera se monitoreó la concentración horaria de $\mathrm{O}_{3}$ desde las $7 \mathrm{~h} 00$ hasta las 22h00. Los datos de la temperatura del bulbo seco y húmedo así como de humedad ambiental del día de monitoreo se obtuvieron de la página web del Servicio Meteorológico Nacional (SMN) (http://smn.cna.gob.mx/ es/pronostico-del-tiempo-pormunicipios). Después de la exposición de los detectores se comparó el color cada uno de ellos con la escala mostrada en la figura 1A y se asignó el color de acuerdo al número de Schönbein. Una vez obtenido el número de Schönbein, junto con el valor de la humedad ambiental, se calculó la concentración de ozono en partes por billón ( $\mathrm{ppb}$ ) con ayuda de la figura $1 \mathrm{~B}$.
A

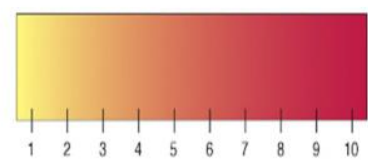

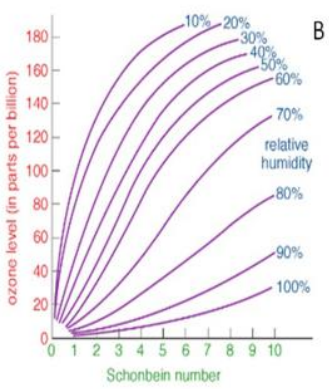

Figura 1. A) Escala de color para calcular el número de Schönbein después de 8 horas de exposición, B) Nivel de ozono ambiental de acuerdo a Walter y Wood (2010).

\section{Resultados y discusiones}

\subsection{Determinación de la concentración de $\mathrm{O}_{3}$ en el Municipio de Mineral de la Reforma}

La coloración que presentaron los detectores después de estar expuestos en el sitio de estudio, se comparó con la tabla de colores del número de Schönbein (Figura 1A). Posteriormente se realizó el cálculo de la concentración de $\mathrm{O}_{3}$, tomando en cuenta la humedad relativa de cada hora de exposición de acuerdo a las concentraciones mostradas en la Figura 1B. En la Tabla 2, se muestran los datos de las concentraciones de $\mathrm{O}_{3}$ del sitio de estudio durante el tiempo de muestreo.

En la Figura 2A se puede observar que las concentraciones de $\mathrm{O}_{3}$ en la zona de estudio mostraron una distribución unimodal a lo largo del día, donde la máxima concentración de $\mathrm{O}_{3}$, de $120 \mathrm{ppb}$, medida fue entre las $12 \mathrm{~h} 00$ y $13 \mathrm{~h} 00$, a una 
temperatura promedio de $18.5^{\circ} \mathrm{C}$ y una humedad relativa entre 16 y $20 \%$. De acuerdo a los criterios establecidos por la normatividad mexicana en la NADF-009-AIRE-2006 (Gaceta Oficial del Distrito Federal, 2006), durante las $12 \mathrm{~h} 00$ y $14 \mathrm{~h} 00$, la calidad del aire en la zona de estudio fue mala y el resto del tiempo fue regular (Figura 2B).

Tabla 2: Concentración de $\mathrm{O}_{3}$ horaria del día 9 de marzo de 2017 en la

\begin{tabular}{cccccc}
\multicolumn{5}{c}{ Colonia Carboneras, Mineral de la Reforma, Hidalgo. } \\
\hline No. muestra & Hora & $\mathrm{Tb}_{\mathbf{s}}{ }^{\circ} \mathrm{C}$ & $\% \mathrm{Y}$ & NSch** & $\begin{array}{c}{\left[\mathrm{O}_{3}\right]} \\
(\mathrm{ppb})^{*}\end{array}$ \\
\hline 1 & $7: 00$ & $10^{\circ} \mathrm{C}$ & $62 \%$ & 1.0 & 19 \\
2 & $8: 00$ & $13^{\circ} \mathrm{C}$ & $51 \%$ & 1.0 & 22 \\
3 & $9: 00$ & $13^{\circ} \mathrm{C}$ & $40 \%$ & 1.0 & 40 \\
4 & $10: 00$ & $14^{\circ} \mathrm{C}$ & $33 \%$ & 1.0 & 58 \\
5 & $11: 00$ & $16^{\circ} \mathrm{C}$ & $26 \%$ & 1.5 & 85 \\
6 & $12: 00$ & $18^{\circ} \mathrm{C}$ & $20 \%$ & 2.5 & 120 \\
7 & $13: 00$ & $19^{\circ} \mathrm{C}$ & $16 \%$ & 2.0 & 120 \\
8 & $14: 00$ & $19^{\circ} \mathrm{C}$ & $27 \%$ & 2.5 & 115 \\
9 & $15: 00$ & $19^{\circ} \mathrm{C}$ & $30 \%$ & 2.5 & 100 \\
10 & $16: 00$ & $18^{\circ} \mathrm{C}$ & $37 \%$ & 3.0 & 80 \\
11 & $17: 00$ & $17^{\circ} \mathrm{C}$ & $43 \%$ & 2.5 & 76 \\
12 & $18: 00$ & $16^{\circ} \mathrm{C}$ & $52 \%$ & 2.0 & 42 \\
13 & $19: 00$ & $13^{\circ} \mathrm{C}$ & $65 \%$ & 1.5 & 19 \\
14 & $20: 00$ & $12^{\circ} \mathrm{C}$ & $75 \%$ & 1.0 & 10 \\
15 & $21: 00$ & $11^{\circ} \mathrm{C}$ & $74 \%$ & 1.0 & 5 \\
16 & $22: 00$ & $11^{\circ} \mathrm{C}$ & $66 \%$ & 1.0 & 11 \\
\hline
\end{tabular}

$\mathrm{T}_{\text {bs: }}$ : Temperatura de búlbo seco, \% Y: Humedad relativa, NSch**: Número de Schönbein.

De acuerdo al reporte del SMN, el índice de radiación (hv) fue de 11 entre las $12 \mathrm{~h} 00$ y $13 \mathrm{~h} 00$ horas del día del muestreo. Esta radiación reaccionó con el $\mathrm{NO}_{2}$ atmosférico, de acuerdo a la ecuación (1), produciendo $\mathrm{O}$ y $\mathrm{NO}$, los cuales pueden a su vez reaccionar de forma natural según las ecuaciones (2) y (3). Lo anterior explica la presencia los altos niveles de $\mathrm{O}_{3}$ en la atmósfera durante ese periodo. Las máximas concentraciones de ozono obtenidas en este trabajo se encuentran dentro del mismo periodo de tiempo de acuerdo a lo reportado en el informe del INECC de la calidad de aire en el 2014. Así mismo, la máxima concentración obtenida en este trabajo (120 ppm) se encuentra dentro de los valores máximos reportados en la zona de Pachuca, Hidalgo, los cuales fueron de 110 y 145 ppb (Tabla 1).

$$
\begin{array}{ll}
\mathrm{NO}_{2}+\mathrm{hv} \rightarrow & \mathrm{NO}+\mathrm{O} \\
\mathrm{O}_{3}+\mathrm{NO} \rightarrow & \mathrm{NO}_{2}+\mathrm{O}_{2} \\
\mathrm{O}+\mathrm{O}_{2} \rightarrow \mathrm{O}_{3} &
\end{array}
$$

En la Tabla 3 se puede observar que las concentraciones de $\mathrm{O}_{3}$, determinadas el día 9 de marzo de 2017 en la Colonia Carboneras del municipio de Mineral de la Reforma, pueden provocar efectos a la salud de las personas como: irritación de nariz y garganta en concentraciones de 50 a 100 ppb y disminución de la agudeza visual, dolores de cabeza, tos, fatiga, sensación de opresión en el pecho y dolor subesternal (en cavidad torácica) en concentraciones de $150 \mathrm{ppb}$. Lo anterior justifica la necesidad de contar con una red de monitoreo más completa, que cubra todas las ciudades del estado, y que se informe oportunamente a la población acerca de las medidas precautorias que deben tomar para proteger su salud.

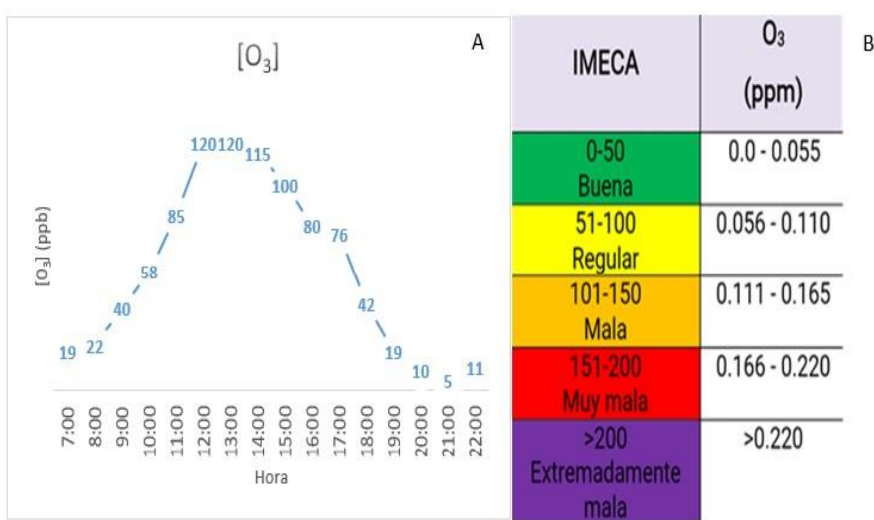

Figura 2: A) Concentración de $\mathrm{O}_{3}$ en el sitio de estudio, B) parámetros de calidad del aire respecto a la NADF-009-AIRE-2006 del 29 de noviembre

\begin{tabular}{|c|c|}
\hline $\mathrm{O}_{3}$ & Efectos a la salud \\
\hline 10 a $15 \mathrm{ppb}$ & Umbral de detección olfativo \\
\hline 50 a $100 \mathrm{ppb}$ & Irritación de la nariz y garganta \\
\hline 150 a $1000 \mathrm{ppb}$ & $\begin{array}{l}\text { Disminución de la agudeza visual, dolores de cabeza, tos, fatiga, sensación de } \\
\text { opresión en el pecho, dolor subesternal }\end{array}$ \\
\hline 1.5 a $2.5 \mathrm{ppm}$ & $\begin{array}{c}\text { Trastornos neurológicos y de coordinación, dificultad de expresión, alteraciones } \\
\text { hematológicas }\end{array}$ \\
\hline 4 a 5 ppm & Edema pulmonar hemorrágico \\
\hline $10 \mathrm{ppm}$ & Coma \\
\hline$>15 \mathrm{ppm}$ & Mortal \\
\hline
\end{tabular}
de 2006.

Tabla 3: Efectos en la salud con concentración de ozono.

\section{Conclusión}

Los datos de las concentraciones de ozono mostraron un comportamiento unimodal, con concentraciones máximas que superan los límites permisibles de este contaminante de acuerdo con la normatividad vigente. Los detectores colorimétricos preparados con una solución de almidón/KI permitieron evaluar de manera sencilla y económica el comportamiento de la concentración de ozono en el sitio de estudio. Esta técnica, a cambio de una menor precisión, proporciona resultados aproximados de la concentración de ozono en el aire, sin necesidad de equipos e instrumentos sofisticados para su aplicación.

\section{English Summary}

\begin{abstract}
Ozone (O3) is present in different layers of the atmosphere: in the stratosphere, it acts as a filter for ultraviolet radiation emitted by the sun; while in the troposphere, it is a harmful pollutant for living organisms, and it is also a precursor gas of greenhouse effect. The objective of this study was to evaluate the hourly variations in the concentration of $\mathrm{O}_{3}$ in the municipality of Mineral de la Reforma, Hidalgo, for this we use colorimetric detectors prepared with a starch / KI solution.
\end{abstract}


The results obtained by the exposure of the detectors to the $\mathrm{O}_{3}$ in the study area showed that the maximum concentration of this gas occurred at $12 \mathrm{~h} 00$, with an average temperature of $18.5^{\circ} \mathrm{C}$ and a relative humidity between 16 and $20 \%$.

Keywords:

Ozone detector, air quality, secondary pollutant, health.

\section{Agradecimientos}

Agradecemos a la Universidad Autónoma del Estado de Hidalgo, especialmente al personal que labora en el Laboratorio de Usos Múltiples, por brindarnos el apoyo, material y equipo necesarios para la elaboración de los sensores colorimétricos.

\section{Referencias}

Ferrer C.J.A., Escalante S.R.I. (2009) Contaminación atmosférica y efectos sobre la salud en la Zona Metropolitana del Valle de México. Economía informa (360), 22-39.

Gaceta Oficial del Distrito Federal (2006) Norma Ambiental para el Distrito Federal NADF-009-AIRE-2006, que establece los requisitos para elaborar el índice metropolitano de la calidad del aire.

INECC, Instituto Nacional de Ecología y Cambio Climático (2015) Informe Nacional de Calidad del Aire 2014. Ciudad de México.

NOM, Norma Oficial Mexicana-020-SSA1-2014. Salud ambiental. Valor límite permisible para la concentración de ozono $\left(\mathrm{O}_{3}\right)$ en el aire ambiente y criterios para su evaluación prefacio.

SMN, Servicio Meteorológico Nacional, recuperado de: http://smn.cna.gob.mx/es/pronostico-del-tiempo-por-municipios, 9 de marzo de 2017.

Vallejo, M., Jáuregui-Renaud, K., Hermosillo, A.G., Márquez, M.F., Cárdenas, M. (2003) Efectos de la contaminación atmosférica en la salud y su importancia en la Ciudad de México. Gaceta Médica Mexicana, 139, 57-63. 